Archives de sciences sociales des religions

124 | octobre - décembre 2003

Varia

\title{
Les Conversions au catholicisme en France : un religieux en mutation?
}

Nicolas de Bremond d'Ars

\section{(2) OpenEdition}

Édition électronique

URL : http://journals.openedition.org/assr/995

DOI : 10.4000/assr.995

ISSN : $1777-5825$

Éditeur

Éditions de l'EHESS

\section{Édition imprimée}

Date de publication : 1 octobre 2003

Pagination : 19-38

ISBN : 2-222-96739-2

ISSN : 0335-5985

\section{Référence électronique}

Nicolas de Bremond d'Ars, "Les Conversions au catholicisme en France : un religieux en mutation? ", Archives de sciences sociales des religions [En ligne], 124 | octobre - décembre 2003, mis en ligne le 10 octobre 2006, consulté le 30 avril 2019. URL : http://journals.openedition.org/assr/995 ; DOI : 10.4000/assr.995 


\section{LES CONVERSIONS AU CATHOLICISME EN FRANCE : UN RELIGIEUX EN MUTATION?}

La société libérale moderne impose aux religions institutionnelles, présentes en Europe depuis des siècles, des mutations dont on ne perçoit pas l'impact à long terme. Si le catholicisme en France est donc en train de vivre pour sa part une « fin du monde » (1), quels sont les signes qui accréditeraient l'hypothèse d'une simple mutation, de grande ampleur certes, là où d'autres annonceraient sa disparition prochaine? Par-delà les manifestations de confiance qui prendraient appui, paradoxalement, sur les faiblesses indéniables du dispositif institutionnel catholique pour envisager un avenir ouvert (2), nous voudrions livrer ici un premier dossier pratique. Il concerne les adhésions adultes au catholicisme.

Le Service National du Catéchuménat (SNC) (3) a mené entre septembre 2001 et janvier 2003 une vaste enquête sur la formation et le devenir des adultes qui sollicitent l'adhésion chrétienne dans le catholicisme. Dans la mesure où il était difficile de repérer d'une façon globale les nouveaux catholiques - ils sont soit intégrés, soit disparus, et ne sont que rarement regroupés en tant que tels - le parti pris méthodologique a été de s'interroger sur les conditions dans lesquelles les candidats sont socialisés au catholicisme. L'hypothèse structurante de ce travail est que les conditions dans lesquelles ils sont socialisés déterminent pour l'essentiel l'avenir auquel ils vont participer. De ce fait, l'enquête a été délibérément orientée en vue d'une collecte massive de données concernant le fonctionnement des catéchuménats en France.

Les résultats de l'enquête manifestent qu'un renouvellement des communautés catholiques par les baptêmes d'adultes succède, pour une part encore modeste mais

(1) Danièle Hervieu-LÉger, Catholicisme: la fin d'un monde?, Paris, Bayard, 2003.

(2) Albert Rouet, Les chances d'un christianisme fragile, Paris, Bayard, 2001.

(3) Le travail de la conférence des évêques de France s'appuie sur les travaux d'experts et sur les structures qui assurent le suivi des orientations. Le Service National du Catéchuménat est le correspondant des différents services diocésains. Aucun de ces services n'a, à notre connaissance, de rôle de gouvernement; il s'agit essentiellement d'études, de collecte de données, de mise à disposition de documents, etc. 
non négligeable, à la classique reproduction par l'admission des enfants. L'intégration dans le peuple catholique de ces nouveaux membres provoquera-t-elle à terme des changements dans l'acclimatation de ce peuple à la modernité ? Sans nous risquer à émettre un pronostic, nous voudrions ici souligner quelques effets potentiels, voire déjà perceptibles, de la présence des catéchumènes. Les catéchuménats sont présents de façon générale depuis quinze ans, soit une demi génération. Leur activité a, d'ores et déjà, contribué à modifier la perception de l'espace de socialité catholique. La paroisse n'est plus l'unique référence de socialisation croyante. Cette modification du rapport à l'espace est sans doute corrélée au profil des nouveaux catholiques: la fluence des appartenances et des réseaux d'adhésion pourrait en effet servir le projet des impétrants marqués par le processus moderne d'individualisation du croire. Il conviendrait de s'interroger, en troisième lieu, sur la transformation des modalités de transmission de la mémoire catholique - rites, pratiques et contenus de croyances. L'examen des lettres de demande de baptême s'avère à cet égard riche d'enseignements.

\section{Le catéchuménat en France : statistiques et organisation}

Les chiffres du catéchuménat n'ont pas été centralisés par le SNC avec la rigueur nécessaire sur une longue période. Le principe de décentralisation qui guide l'exercice de l'autorité dans l'Église catholique en est largement responsable : seul l'échelon diocésain a valeur d'obligation. Les chiffres que nous donnons sont, jusqu'à la fin des années quatre-vingt-dix, des estimations.

En 1980, on comptabilisait environ 2800 personnes adultes en démarche catéchuménale : préparation au baptême, à la première eucharistie, à la confirmation. Dix ans après, en 1991, le chiffre était estimé à 5 643, puis montait rapidement pour atteindre environ 10000 personnes en 1995. Sur ce dernier chiffre, on comptabilisait environ 3500 baptêmes prévus. Depuis cette date, les baptêmes proprement dits oscillent entre 2200 (en 1998) et 2500 (en 1999). Le chiffre est stabilisé aux alentours de 2300 pour les dernières années. Il est cependant difficile d'ajouter les futurs confirmés (2 000 par an), parce qu'on ne dispose pas d'éléments permettant de déterminer s'il s'agit de néophytes ayant été récemment baptisés ou de recommençants.

Si l'on comptabilise le nombre de catéchumènes en démarche, sachant que la durée moyenne du catéchuménat est de deux à trois ans, on peut estimer qu'environ 50000 personnes (dix mille par an) ont fréquenté en dix ans l'Église catholique pour y adhérer.

\subsection{Conditions de l'enquête}

Le déroulement de l'enquête a bénéficié de la structure de fonctionnement du SNC. Relié à l'ensemble de la France, ce service a permis un contact avec l'ensemble des diocèses, et tout particulièrement avec ceux qui ont chaque année de nombreux catéchumènes. Trois éléments constituent l'essentiel des données. Une 
première phase a consisté à lancer un Questionnaire National (QN) qui couvrirait le maximum du territoire français. Le principe d'une enquête rapide à laquelle les responsables pouvaient répondre sans difficulté a été privilégié. Il suffisait, dans de nombreuses questions, de cocher les cases qui correspondaient à la réponse, et la parole libre a été minimisée. Les questions ouvertes, présentes dans le questionnaire, sont de ce fait peu fréquentées par ceux qui y répondent. Le taux de retour des questionnaires envoyés au cours de la première phase atteste du choix judicieux qui a été ainsi fait.

La deuxième phase de l'enquête porte sur les accompagnateurs. Initialement, il avait été prévu d'enquêter sur les catéchumènes directement, mais les réponses qui ont été apportées aux premiers questionnaires adressés aux responsables de catéchuménat locaux ont conduit à privilégier l'approche par les accompagnateurs (4). Malgré tout, si les Questionnaires Accompagnateurs (QA) ne sont pas un échantillon statistiquement fiable, ils constituent la meilleure base de données disponible à ce jour. Ils fournissent un instantané de l'accompagnement catéchuménal en France. La convergence importante des réponses, par-delà la dispersion des provenances géographiques, tempère l'éventuel discrédit que l'on pourrait, en toute rigueur, jeter sur ce questionnaire.

Une troisième phase de l'enquête porte sur l'expression des catéchumènes euxmêmes. Les responsables ayant obligeamment proposé de fournir les «lettres de demande de baptême », il a été décidé d'en faire une analyse poussée. Ces lettres sont écrites par les catéchumènes en fin de formation lorsqu'ils demandent officiellement à être baptisés. Nous avons eu accès à 250 lettres en provenance de six diocèses différents, écrites entre 1999 et 2002. Grâce à elles, nous pouvons comparer les renseignements issus des responsables locaux, ceux émis par les accompagnateurs et les effets sur le langage de la foi qui se donne sous la plume des sujets de la conversion.

\subsection{Glossaire et définitions}

Catéchumène : mot d'origine grecque signifiant «celui en qui résonne » la parole de Dieu. Ce terme désigne précisément la personne qui appartient à un groupe de préparation aux trois sacrements de l'initiation chrétienne, le baptême, la confirmation, l'eucharistie. Le catéchumène est considéré comme appartenant à l’Église, de manière spéciale (canon $205 \S 1$ ).

Sacrement: acte liturgique qui manifeste et communique aux hommes «le mystère de la communion du Dieu amour, Un en trois personnes (5). » Baptême et confirmation sont uniques et confèrent un "caractère indélébile » à celui qui les reçoit (dont l'identité chrétienne); l'eucharistie (ou communion) est réitérable et le

(4) Poser la question de la validité statistique de ce Questionnaire Accompagnateurs n'est malheureusement pas à l'ordre du jour. Dans la mesure où le retour dépend a) de la qualité de pénétration du Questionnaire National (on pouvait déclarer vouloir poursuivre la collaboration ou non), b) de la qualité de mobilisation par les responsables diocésains et locaux, et c) de la bonne volonté des accompagnateurs eux-mêmes, on voit que les centaines de questionnaires remplis ne peuvent en aucun cas prétendre à la représentativité.

(5) Catéchisme de l'Église catholique, Paris, Mame-Plon, 1992, no 1118. 
catholique a injonction de communier au moins une fois l'an. En général, le baptême est célébré au cours de la Veillée pascale (nuit précédant le jour de Pâques), dans la paroisse ou dans l'église cathédrale (plus rarement). Habituellement la confirmation et l'eucharistie sont célébrés au cours de la même liturgie, mais quelques diocèses diffèrent parfois la confirmation pour la réserver à l'évêque.

Appel décisif : à l'issue de la préparation, le catéchumène écrit à l'évêque de son diocèse pour demander le baptême, la confirmation et l'accès à l'eucharistie. L'évêque répond par l'appel décisif qui manifeste son acception de la requête. L'appel est célébré au cours d'une liturgie unique pour tout le diocèse.

Étapes de baptême : le parcours du catéchumène est jalonné d'étapes liturgiques, qui peuvent être autant d'étapes d'intégration à la communauté locale. Il s'agit de l'entrée en catéchuménat, des scrutins (en nombre variable), de la redditio du Credo (= profession de foi), de la traditio du Notre Père, etc.

Recommençant : tout chrétien adulte ayant reçu (dans son enfance ou adolescence) tout ou partie des sacrements de l'initiation chrétienne ainsi qu'une instruction (catéchisme), qui a interrompu pendant un temps indéterminé sa pratique, et qui cherche à se réinitier. Parfois il s'agit d'un adulte ayant été baptisé enfant et n'ayant eu aucune instruction.

Néophyte : nouveau chrétien, ayant été admis aux trois sacrements de l'initiation. Dans la formation traditionnelle, les néophytes devaient poursuivre une catéchèse de façon à comprendre ce qu'ils avaient appris : le savoir n'est efficace que s'il est mis en pratique.

\section{L'environnement catholique en France}

L'apparition diffuse dans les paroisses de personnes réclamant l'adhésion chrétienne (6) a modifié progressivement la manière dont fonctionne l'espace public catholique. La nécessité de transmission de la foi était jusque-là comprise très majoritairement dans un rapport collectif. La paroisse (ou son extension plus ou moins indépendante, l'aumônerie (7)) accueille des enfants et des jeunes confiés par leurs parents. Inscrits dans une équipe, ils sont d'emblée en interaction avec l'Institution sur le mode collectif.

Or l'arrivée d'individus isolés ne permet pas, en première instance, de constituer un groupe. Deux stratégies sont alors mises au point. D’une part les autorités locales en réfèrent au-delà de la paroisse, aux paroisses voisines, dans le doyenné ou le secteur, et au diocèse. Les candidats sont alors formés en équipe de secteur (par exemple), et pris en charge parfois de façon complémentaire par les instances diocésaines.

(6) La rigueur voudrait que l'on parle d'adhésion «catholique ». Or le baptême pratiqué dans le catholicisme n'est que « chrétien » puisque les catholiques admettent l'adhésion chrétienne dans d'autres confessions non catholiques (orthodoxes, protestants, ...). Il n'existe donc pas de " baptême catholique ».

(7) Nous parlons «d'extension » pour signifier que tous les actes de catholicité sont le monopole de la paroisse. On ne peut baptiser quiconque dans une aumônerie sans l'autorisation du curé du lieu, et le baptême est noté sur les registres paroissiaux. 
Corollairement à cet élargissement de l'espace, une relation d'apprentissageaccompagnement est organisée avec un accompagnateur local, qui devient ainsi le référent privilégié du catéchumène. L'environnement catholique paroissial est donc soumis à trois tensions critiques: la mouvance des territoires et des niveaux d'appartenance catholique, l'individualisation de la transmission et la modification du dispositif d'autorité.

\subsection{Mouvance des territoires}

L'enquête sur le catéchuménat déploie les nouvelles structures de l'institutionnalité catholique en France. Par-delà les regroupements de paroisses opérés dans la grande majorité des diocèses commence à se mettre en place un nouveau rapport à l'espace. Le diocèse devient une réalité pertinente pour les acteurs.

L'examen des lettres écrites par les catéchumènes pour l'appel décisif a été pratiqué par diocèse. La recherche de zones culturelles n'a pas établi de regroupements significatifs. Les contenus sémantiques, en revanche, fournissent l'indice d'une identité diocésaine. Chaque diocèse comporte un profil particulier de contenus du croire, légitimant par là une régulation à son niveau et non au niveau local (secteur ou paroisse).

L'échelon local demeure cependant l'échelon pertinent d'ancrage et de transmission. Les célébrations qui ponctuent le parcours du catéchumène ne sont pas tenues en-dehors des paroisses. Pour autant, la constitution des équipes de formation échappe, nous l'avons dit, à l'autorité curiale.

Le local est une catégorie qui s'est transformée. Nous avons pu identifier 44 dossiers classés en zone urbaine qui mentionnaient l'existence de plusieurs clochers, et 62 dossiers qui annonçaient un fonctionnement en secteur (ou doyenné). Ajoutés aux dossiers classés en milieu rural, dont la grosse majorité mentionne plusieurs clochers, nous avons un total d'environ 160 lieux (soit $45 \%$ de l'ensemble) pour lesquels paroisse ne rime plus avec clocher, ni communauté chrétienne avec l'échelon communal. L'appartenance catholique ne se réfère plus immédiatement à un découpage géographique, mais à une unité d'action pastorale, qui varie d'un diocèse et d'une région à l'autre.

Les séances de catéchèse aussi bien que la coordination sont assurées par une instance qui déborde la paroisse. Le secteur pastoral devient l'aire d'influence et d'autorité régulatrice : si le curé continue d'être maître chez lui, les dispositifs sont au minimum suggérés par l'équipe coordinatrice, et dans nombre de cas organisés par elle.

Le paysage qui se dessine là révèle, plus souterrainement, le basculement des logiques ecclésiales qui, de rurales, redeviennent prioritairement urbaines. Deux faits confirment cette évolution.

Une première différenciation est inscrite dans la durée d'existence des catéchuménats. En zone urbaine, ils existent à $66 \%$ depuis plus de 10 ans, contre $18 \%$ en rural; si on prend en compte l'existence depuis plus de 5 ans, on obtient au total $86,6 \%$ en urbain et $52,8 \%$ en rural. Les secteurs urbains connaissent donc le catéchuménat depuis plus longtemps, ce qui tendrait à montrer que les structures de l'ecclésialité rurale sont en position d'apprentissage par rapport à l'ecclésialité 
urbaine. Cela signifie nettement que le catéchuménat est une initiative urbaine, et que le rural prend modèle sur l'urbain.

L'enquête (QA) s'est enquise de la demande faite ou non aux catéchumènes (débutants, puis ceux ayant franchi des étapes) de "participer à la messe du dimanche» et de 《participer à des activités (paroissiales ou autres)». Les réponses, rétablies en pourcentage, ne sont sans doute pas très significatives, car la dispersion possible des réponses s'applique à un échantillon trop réduit en ce qui concerne le rural (56 dossiers disponibles sur le sujet). À première vue, il ne semblerait pas que la distinction rural-urbain soit opératoire. En ce qui concerne la messe, pour les débutants il est demandé dans de semblables proportions (19\% en urbain, $23 \%$ en rural), pour les accompagnateurs l'écart est un peu plus élevé (78\% en urbain, $62 \%$ en rural) (8). Les résultats sont équivalents en ce qui concerne la participation aux activités.

La prévalence du modèle urbain s'apprécie mieux dans son rapport à la modernité lorsqu'on le met en rapport avec le contenu de la formation catéchuménale.

\subsection{De la communautarisation à l'adhésion individualisée}

Le système de formation organisé dans la plupart des catéchuménats comprend les deux volets traditionnels : un accompagnateur personnel et une équipe de catéchèse. La complémentarité des deux modes est censée tenir ensemble la personnalisation indispensable de la formation et l'insertion dans une histoire collective. Dans la mesure où les candidats arrivent individuellement, il serait raisonnable de supposer que la socialisation au catholicisme constitue l'objectif principal de l'itinéraire. Or, plusieurs aspects de l'enquête convergent dans le sens inverse, comme si les catéchumènes amenaient avec eux la tendance individualisante des adhésions religieuses; à moins qu'ils n'aient fait que la réveiller chez leurs accompagnateurs ... L'intensité de la formation et les thématiques qui y sont abordées orientent les candidats vers une perception moins communautaire qu'individualisée de l'adhésion.

\subsubsection{Intensité et individualisation sont corrélées}

Plusieurs figures de formation sont disponibles, que l'on peut classer en termes d'intensité et en termes de degré de socialité. D'un côté se trouvent les formations qui privilégient la rencontre individuelle, par opposition à des formations qui privilégient le collectif; de l'autre se trouvent les formations à forte intensité relationnelle, par opposition à des formations de faible intensité :

(8) Il convient cependant de tenir compte d'un accès difficile à la messe dans le monde rural ; les questionnaires signalent une messe par dimanche, voire moins. 


\begin{tabular}{|c|l|l|l|l|}
\hline $\begin{array}{r}\text { Réunions } \\
\text { Rencontrestives }\end{array}$ & \multicolumn{1}{|c|}{$<\mathbf{8}$} & $\mathbf{8}$ à $\mathbf{1 2}$ & $\mathbf{1 2}$ à 16 \\
individ.
\end{tabular}

Tableau 1 : typologie des rencontres annuelles proposées dans le cadre d'une formation catéchuménale (9).

Les catéchumènes suivent une formation majoritairement individuelle, comme en témoigne le tableau ci-dessous, et son intensité est forte :

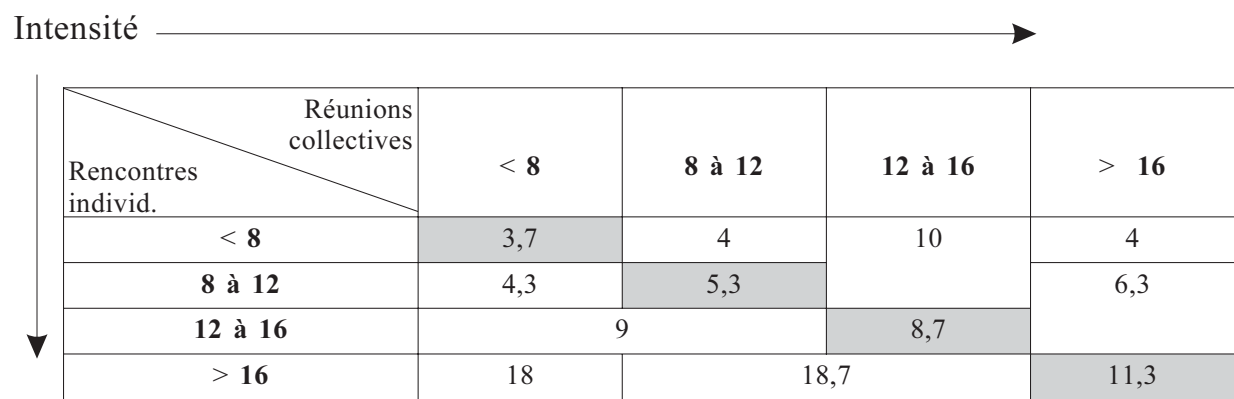

Tableau 2 : Ensemble des réponses exploitables rural + urbain exprimées en \%.

La domination de l'individuel n'est guère contestable. $\mathrm{Si}$, à intensité soutenue, l'équilibre est trouvé entre réunions collectives et rencontres individuelles, la tendance est nettement favorable à l'individuel lors de l'accroissement d'intensité : $36,7 \%$ des catéchuménats fonctionnent avec une insistance sur la formation individuelle, contre $10,3 \%$ de type collectif.

Une confirmation en est donnée par les accompagnateurs eux-mêmes. L'accompagnateur n'est pas un novice : $76 \%$ de ceux qui ont répondu n'en sont pas à leur premier accompagnement (30\% en ont plusieurs simultanément). Cela

(9) Le classement des réunions et rencontres a été fait en fonction du rythme habituel d'une vie paroissiale, qui se calque plus ou moins sur l'année scolaire. L'expérience montre qu'on peut tabler sur 8 réunions mensuelles d'octobre à juin (neuf mois moins un en raison des congés scolaires). 12 et 16 sont donc des divisions commodes qui ajoutent $1 / 2$ et 1 année; sur deux années de formation, par exemple, il peut y avoir raisonnablement 16 réunions ou rencontres. 
peut signifier ou bien la constitution d'une caste de semi-professionnels de l'accompagnement ou, plus prosaïquement, l'absence de personnes compétentes et disponibles (10). De ce fait, on privilégierait l'expérience.

Il/elle rencontre son catéchumène à raison d'une fois par mois $(70 \%$ des cas $)$, tout en étant prêt(e) à lui accorder plus : 19\% déclarent le rencontrer de façon quasi hebdomadaire. Son travail d'accompagnement n'est lui-même que peu confronté à celui des autres accompagnateurs, ce qui renforce la transmission de type individuel. Il n'y a en effet que 2 à 4 rencontres d'accompagnateurs dans l'année (64\% des cas), voire pas du tout dans $8 \%$ des catéchuménats. Mais $28 \%$ des dossiers mentionnent tout de même plus de 4 rencontres dans l'année.

\subsubsection{Une thématique individualisante}

Le contenu des entretiens entre accompagnateur et catéchumène est du ressort privé. On dispose cependant de réponses qui lèvent un coin du voile. Les thématiques présentes dans les entretiens ont été classées par les accompagnateurs sur une échelle de 1 à 9, du plus au moins étudié. Même en accordant une importance modérée à la dispersion des réponses centrales, les écarts présentés dans le tableau 3 sont très significatifs.

Les thèmes à connotation personnelle sont les plus fréquemment abordés. La vie intérieure ( vie spirituelle») est privilégiée par rapport à la vie extérieure ( «éthique »), la relation personnelle au divin («Évangile, Jésus-Christ») l'est par rapport à la relation au groupe («histoire de l'Église»). Les interrogations des catéchumènes portent donc, en phase avec leur époque, sur la manière dont se constitue la croyance, entendue au sens restreint de l'adhésion personnelle. La pratique entendue au sens public concerne, dans le tableau 3, la "vie ecclésiale » (liturgie, prière, sacrements), l'éthique et les engagements (charité, solidarité, syndicalisme, etc.) : bien que le classement soit à prendre avec précaution, il apparaît nettement que ce sont des contenus de second rang.

\begin{tabular}{|l|c|c|c|c|c|c|c|c|c|}
\hline & $\begin{array}{c}\text { Évang. } \\
\text { JC }\end{array}$ & $\begin{array}{c}\text { Vie spiri } \\
\text { tuelle }\end{array}$ & $\begin{array}{c}\text { Bible en } \\
\text { général }\end{array}$ & Credo & $\begin{array}{c}\text { Vie } \\
\text { ecclés. }\end{array}$ & $\begin{array}{c}\text { Engage- } \\
\text { ments }\end{array}$ & Mal & Éthique & $\begin{array}{c}\text { Hist. de } \\
\text { ''Église }\end{array}$ \\
\hline 1 & 69,7 & 15,6 & 4,9 & 5,2 & 1,3 & 0,7 & 1,6 & 0,3 & 0,3 \\
\hline 2 & 17,2 & 29,6 & 21,5 & 14,3 & 4,6 & 6,2 & 3,9 & 2,3 & 0,3 \\
\hline 3 & 7,2 & 25,1 & 16 & 17,3 & 12,7 & 10,7 & 5,5 & 3,3 & 1,6 \\
\hline 4 & 2,6 & 12,7 & 13,4 & 17,6 & 24,1 & 11,4 & 10,4 & 7,5 & 1,3 \\
\hline 5 & 2,3 & 5,9 & 16,6 & 18,2 & 20,8 & 14 & 10,1 & 12,4 & 1,6 \\
\hline 6 & 1 & 4,6 & 8,8 & 13,7 & 16,9 & 17,6 & 22,1 & 12,1 & 4,9 \\
\hline 7 & - & 3,9 & 8,8 & 7,8 & 12,7 & 15,6 & 16,9 & 22,8 & 10,1 \\
\hline 8 & - & 2,6 & 7,8 & 3,6 & 6,2 & 14,3 & 18,6 & 28 & 17,9 \\
\hline 9 & - & - & 2,3 & 2,3 & 0,7 & 9,4 & 10,7 & 11,4 & 61,9 \\
\hline médian & 1 & 2,2 & 3,6 & 3,75 & 4,35 & 5,4 & 5,8 & 6,5 & 9 \\
\hline
\end{tabular}

Tableau 3 : Fréquence des notes attribuées à chaque thème en \%; en grisé, note majoritaire.

(10) Le diocèse de Paris a explicitement demandé en 2003 que les accompagnateurs disponibles dans les paroisses offrent leurs services dans tout le diocèse, sans se limiter à leur communauté ; nous sommes bien dans le cadre d'une professionnalisation. 
Faut-il incriminer les accompagnateurs qui orienteraient délibérément les discussions dans le domaine intimiste de la vie spirituelle, ou bien les contenus répondent-ils aux requêtes des candidats au baptême ? L'enquête ne permet pas de le dire. Compte tenu de l'ancienneté des accompagnateurs et de la jeunesse des catéchumènes, nous pencherions pour la deuxième hypothèse.

La mise à l'écart de la vie éthique et de l'histoire de l'Église complète le tableau. Ces deux thèmes représentent les conditions d'une transmission de la mémoire collective. L'adoption des contenus et pratiques éthiques, définis comme les valeurs communes, et l'insertion dans une histoire collective dont on connaît les difficultés sur la scène publique (cf. les actes de repentance concernant la Seconde Guerre mondiale, par ex.) sont les lieux clés de l'intégration. Dans quelle mesure les catéchumènes seront-ils intégrés s'ils demeurent en contradiction, au moins tacite, avec la mémoire et les pratiques communes? La conciliation entre l'adhésion individuelle et l'entrée dans une tradition religieuse instituée trouve ici sa limite. Il n'est pas surprenant de relever alors dans l'enquête la perception d'obstacles à l'intégration des néophytes dans les communautés catholiques.

\subsubsection{Des obstacles à l'adhésion?}

Le renouvellement des populations croyantes pratiquantes en catholicisme se fera-t-il, pour partie, grâce aux nouveaux convertis ? L'ambivalence du dispositif de socialisation que nous venons de décrire produit ses effets sur l'adhésion. Deux indicateurs émergent dans l'enquête, pour suggérer que tous les obstacles ne sont pas levés par l'émotionalité bien attestée de la prise en charge catéchuménale. Ils se complètent : le premier tente de mesurer indirectement la capacité d'accueil des communautés chrétiennes, le second décrit la perception subjective des accompagnateurs.

La mesure de la capacité d'accueil des communautés (souvent des paroisses) s'appuie sur la présence attestée de groupes de nouveaux membres, néophytes et recommençants.

Précisons tout d'abord que le phénomène des recommençants est très nettement inférieur en valeur absolue à celui des catéchumènes. Tout se passe comme si les sacrements de l'initiation chrétienne (baptême, eucharistie et confirmation) constituaient un objectif. Les recommençants, ayant dans leur grande majorité reçu ces sacrements, n'ont pas besoin de se présenter.

L'enquête montre que la capacité d'accueil des lieux catéchuménaux ne préjuge en rien d'un accueil d'éventuels recommençants (tableau 4 ci-après). L'accroissement du nombre de catéchumènes ne signifie pas un accroissement corrélatif du nombre de recommençants. En revanche, il n'y a de recommençants que là où un catéchuménat existe. Il semble donc que les recommençants sont en quelque sorte à la remorque des catéchumènes. Toutefois, compte tenu des objectifs de l'enquête, il n'est pas exclu que les lieux où se trouvent explicitement des recommençants soient différents des lieux de catéchuménat.

Comme pour les recommençants, la présence de néophytes ne se déduit pas de la présence d'un catéchuménat. Lorsque l'on passe de 1-3 catéchumènes par an à plus de 3, c'est-à-dire d'un catéchuménat existant simplement chaque année à un catéchuménat abondant, le nombre de groupes de néophytes diminue (132 dans un cas, 69 dans l'autre). L'accroissement du nombre de catéchumènes ne conduit pas 
automatiquement à une augmentation de néophytes ; on pourrait même soutenir le contraire, puisqu'il y a 96 mentions de néophytes lorsque le catéchuménat réunit 1 ou 2 personnes de temps en temps et seulement 69 lorsqu'il en réunit plus de 3 par an !

La norme semble être d'un néophyte de temps en temps, voire pas du tout (252 cas sur 297). Elle n'est pas corrélée, dans cette catégorie non plus, à la taille du catéchuménat, puisque les néophytes semblent plus nombreux quand le catéchuménat est faible.

Il n'y a donc pas de lien entre la taille du catéchuménat et le nombre de néophytes se retrouvant dans un groupe spécifique par la suite.

On peut en conclure que le catéchuménat, dans son ensemble, ne vise pas à produire une suite. Lorsque celle-ci existe, sous forme d'individus qui se présentent un par un semble-t-il, elle doit obéir à des logiques qui ne sont pas liées au fonctionnement du catéchuménat, mais à d'autres demandes, certainement propres aux personnes ou aux communautés chrétiennes que celles-ci ont fréquentées et qui leur donneraient envie de poursuivre.

La question de l'accueil trouve un éclairage nouveau. Ou bien, en effet, face à une demande spirituelle, les paroisses ne sont pas accueillantes, et les nouveaux chrétiens estiment avoir atteint leur objectif. Dans cet ordre d'idées, l'intégration des néophytes peut se passer plus vite que prévu, auquel cas les néophytes seraient en quelque sorte des 'retardataires' ou des 'redoublants'.

À supposer toutefois que le principe d'une poursuite de la formation au-delà de l'initiation catéchuménale soit un objectif des responsables de catéchuménat, il faut interroger cette initiation qui ne permet pas d'atteindre l'objectif. Il n'y aurait pas de néophytes parce que le type de formation qui est délivré aux catéchumènes n'implique pas que l'identité catholique passe par la participation à un groupe de néophytat.

\begin{tabular}{|c|c|c|c|c|c|c|c|c|c|c|c|c|c|}
\hline \multirow{2}{*}{$\begin{array}{l}\text { Recom. } \\
\text { Néoph } \\
\downarrow\end{array}$} & \multicolumn{4}{|c|}{ Catéchumènes 1 ou 2} & \multicolumn{4}{|c|}{ Catéchumènes 1 à $3 / a n$} & \multicolumn{4}{|c|}{ Catéchumènes 3/an } & \multirow[b]{2}{*}{ tota } \\
\hline & $\begin{array}{l}0 \\
\mathrm{a} \\
\end{array}$ & $\begin{array}{c}1 \text { ou } \\
2 \\
\mathrm{~b}\end{array}$ & $\begin{array}{c}1 \text { à } \\
3 / \mathrm{an} \\
\text { (c) }\end{array}$ & $\begin{array}{c}>3 \\
\mathrm{~d}\end{array}$ & 0 & $\begin{array}{c}1 \text { ou } \\
2 \\
\mathrm{f} \\
\end{array}$ & $\begin{array}{c}1 \text { à } \\
3 / \text { an } \\
(\mathrm{g})\end{array}$ & $\begin{array}{c}>3 \\
\mathrm{~h}\end{array}$ & 0 & $\begin{array}{c}1 \text { ou } \\
2 \\
j\end{array}$ & $\begin{array}{c}1 \text { à } \\
3 / \text { an } \\
\text { (k) }\end{array}$ & $\begin{array}{c}>3 \\
1\end{array}$ & \\
\hline $\begin{array}{l}0 \\
\mathrm{a}\end{array}$ & 5,4 & 6 & - & 0,3 & 3 & 6,4 & 3,7 & - & - & 1,7 & 2,7 & 1 & 30,2 \\
\hline $\begin{array}{l}1 \text { ou } 2 \\
b \text { ' }\end{array}$ & 4,4 & 13,8 & 1 & - & 1,3 & 18,5 & 3,4 & 1,7 & & 5 & 3,4 & 2 & 54,4 \\
\hline 1 à $3 c^{\prime}$ & - & 0,3 & 0,3 & - & 0,7 & 2,7 & 2 & 1,3 & - & 1 & 3,7 & 2 & 14,1 \\
\hline$>3 \mathrm{~d}^{\prime}$ & - & 0,3 & - & - & - & - & - & - & - & - & 0,7 & 0,3 & 1,3 \\
\hline Total & 9,7 & 20,5 & 1,3 & 0,3 & 5 & 27,5 & 9,1 & 3 & - & 7,7 & 10,4 & 5,4 & 100 \\
\hline
\end{tabular}

\section{Tableau 4 : Classement des questionnaires ayant manifesté la présence simultanée de néophytes, recommençants et catéchumènes. Données en \%. En grisé, cases en incohérence.}

Le tableau 4 combine les données portant sur néophytes et recommençants avec la présence de catéchumènes. Sa lecture est un peu malaisée, mais fournit d'utiles précisions. 
Premier constat: les néophytes n'apparaissent jamais nombreux, bien qu'au total plus des deux tiers des lieux mentionnent la présence de néophytes, ce qui est considérable !

Ces néophytes se présentent là où il y a également des recommençants. Concomitamment, là où manquent les recommençants (colonnes 0) manquent aussi les néophytes. Mais on peut mesurer le déplacement du nombre de néophytes en lien avec l'augmentation du nombre de catéchumènes et de recommençants.

$\mathrm{Si}$ on établit un rapport entre les catéchuménats où il n'y a que $\{0\}$ ou $\{1$ ou 2$\}$ recommençants et ceux où il y en a $\{1$ à 3$\}$ ou plus, on voit que le nombre de néophytes croît proportionnellement. La proportion entre \{recommençants + néophytes $\}$ et catéchumènes est de $\mathbf{1 3 , 4}$ pour les catéchuménats à $\{1$ ou 2$\}$ (11); de $\mathbf{2 , 8}$ pour les catéchuménats à $\{1-3\}$ par an (12); de $\mathbf{0 , 5}$ pour les catéchuménats $\{>3\}$ (13). Le résultat est clair: il y a proportionnellement d'autant plus de néophytes et recommençants conjointement qu'il y a de catéchumènes. Cet indicateur mesure donc, en quelque sorte, la vitalité d'un lieu d'accueil, et donne très schématiquement les chances pour un catéchumène accomplissant son initiation de poursuivre une vie chrétienne spécifique par la suite.

En milieu rural, une étude similaire conduit aux résultats suivants :

a) il y a peu de néophytes en monde rural : $40 \%$, là où il y en a $70 \%$ en monde urbain ;

b) globalement, il n'y a pas non plus de recommençants : pas du tout pour $37,5 \%$, $48 \%$ de façon épisodique ;

c) mais à l'inverse, là où il y a des catéchumènes, il y a des recommençants : on trouve de 1 à 3 recommençants par an plus facilement là où il y a de 1 à 3 catéchumènes par an que là où il n'y en a que 1 ou 2 de temps en temps $(8,3$ contre $4,2 \%)$.

On pourrait conclure, donc, sur la base de ces données, que les néophytes repérés dans les lieux de vie catholique existent ni plus ni moins que les recommençants. Ce qui signifie que l'existence des néophytes ne dépendrait pas tant du prolongement d'une formation à l'exemple du catéchuménat, mais bien plutôt de la qualité d'accueil des lieux ecclésiaux. Si un lieu est accueillant, il verra recommençants et néophytes.

Le deuxième indicateur qui va dans le sens de ce que nous cherchons est extrait des réponses fournies par les accompagnateurs eux-mêmes lorsqu'ils sont interrogés sur les facilités ou difficultés d'intégration des néophytes.

Il est intéressant de noter que les prises de position publiques de l'Église entendue ici dans son versant institutionnel et dogmatique (et le mode de déploiement de la vie chrétienne par les «vieux catholiques ») constituent l'obstacle le plus couramment ressenti par les accompagnateurs à propos des néophytes. Ce qui

(11) Somme des cases ab'+bb'+bc'+bd' divisée par cb'+cc'.

(12) $\mathrm{eb}$ ' $+\mathrm{ec}$ ' $+\mathrm{fb}$ ' $+\mathrm{fc}$ ' divisé par $\mathrm{gb}$ ' $+\mathrm{gc}$ ' $+\mathrm{hb}$ ' $+\mathrm{hc}$ '.

(13) Les chiffres se lisent ainsi : "il y a 13,8 fois plus de lieux qui ont \{0 ou 1 à 2 recommençants ET des néophytes $\}$ que de lieux où l'on trouve \{1 à 3 et 3 recommençants ET des néophytes\}, quand le catéchuménat est faiblement présent"; “il y a deux fois moins de lieux qui ont $\{0$ ou 1 à 2 recommençants ET des néophytes $\}$ que de lieux où l'on trouve \{1 à 3 et 3 recommençants ET des néophytes $\}$ quand le catéchuménat est très vivant". 
conduirait à soupçonner l'existence d'un décalage important entre les contenus de croyance, par lesquels se structure l'adhésion religieuse du catéchumène, et les modes de socialisation possibles de ces contenus. Une première hypothèse peut être émise quant à la fiabilité des accompagnateurs eux-mêmes : ne seraient-ils pas, eux aussi, des individus particulièrement sensibilisés, de par leur expérience subjective propre, aux frontières du groupe ? On ne peut écarter le problème de leur recrutement, et de leur rapport au groupe des "pratiquants réguliers », bien intégrés ; peut-être qu'un accompagnateur est aussi en difficulté avec ce groupe dominant ...

Nous n'avons pas été en mesure de lever l'ambiguïté de la déclaration : les accompagnateurs qui répondaient au questionnaire exprimaient-ils leur propre malaise (42\% disent que les communautés locales font obstacle) ou bien une réalité vécue par les catéchumènes ? Dans les deux cas est en cause la qualité de relation entre ceux qui accueillent et les communautés elles-mêmes. Si on considère en effet que le converti pénètre dans un réseau social, alors son accueil et la durée de sa présence dépendent de la qualité du réseau communicationnel (et du contenu, en second lieu) plus que de la relation à un des pôles qui composent le réseau. Que le pôle accueillant entretienne des relations délicates avec le groupe dominant et le converti ne peut pas s'insérer.

Il semble donc exister un hiatus entre la tendance individualisante de la formation chrétienne et la nécessité de rejoindre un réseau relationnel. La complémentarité des modes de formation chrétienne semble échouer à prendre en charge les aspirations à la reconnaissance de chaque personne dans sa singularité, pour les mener à leur terme ecclésial : l'ouverture à l'universel par l'insertion dans un réseau relationnel (14).

Les candidats au baptême le perçoivent-ils ? Comment retranscrivent-ils les données qu'ils ont acquises au cours de leur longue préparation ? Autant d'interrogations auxquelles les lettres rédigées pour l'appel décisif permettent de répondre, au moins partiellement.

\section{3. Être socialisé catholique, ou devenir croyant?}

L'évaluation de la dynamique induite par l'adhésion croyante au catholicisme ne peut faire l'économie de la réception des discours et pratiques de catéchuménat. Changer de religion, acquérir une identité religieuse, devenir croyant: autant d'expressions qui traduisent la difficulté de rendre compte du phénomène de la conversion.

« Dans la perspective sociologique, la conversion représente un arrachement à un milieu social déterminé et l'adhésion à une communauté nouvelle.[...] À l'inverse, il faut remarquer qu'un des mobiles les plus puissants de conversion

(14) Catholique : « Le mot 'catholique' signifie "universel” dans le sens de « selon la totalité » ou « selon l'intégralité » Catéchisme de l'Église catholique, op. cit. On peut y adjoindre la déclaration suivante du concile Vatican II : « L'Église du Christ est vraiment présente en tous les légitimes groupements locaux de fidèles qui, unis à leurs pasteurs, reçoivent, dans le Nouveau Testament, eux aussi, le nom d'Églises (...)» Constitution Lumen Gentium 13. 
réside dans l'attraction qu'exerce la communauté d'accueil, par l'atmosphère de charité ou de charisme qui peut y régner (...) (15). » La définition de la conversion comprend en outre un volet éthique : «D'une façon générale, ce passage d'une communauté à une autre s'accompagne de scrupules moraux (...) (16)», qui portent sur la recomposition du système de valeurs. La modification profonde du système de référence éthique n'est pas perçue comme telle par les accompagnateurs; les lettres de demande de baptême des catéchumènes le confirment clairement. Il est donc très hasardeux d'appliquer le vocabulaire de la conversion de façon uniforme à l'adhésion croyante des catéchumènes actuels au catholicisme. Le refoulement de la formation à l'éthique catholique, manifesté aussi bien dans les thèmes de discussions que dans les obstacles ressentis au sujet de l'Église (prise en tant qu'Institution) ne laissent pas de doute.

Danièle Hervieu-Léger souligne trois figures de la conversion dans les sociétés modernes (17) :

a) l'individu qui change de religion. Son récit s'articule alors autour d'un rejet de la religion d'origine qui est, semble-t-il, incapable de prendre en charge les vrais problèmes des hommes. L'intégration dans une communauté qui prend en compte les aspirations propres de la personne est le versant ensoleillé du récit. Ce type de conversion comprend une composante nette de protestation socioreligieuse ;

b) l'individu qui découvre la religion alors que son univers s'en était passé jusquelà. Il «pose ses valises » dans une religion - ici le catholicisme - après un parcours plus ou moins long, plus ou moins sinueux. La soif de vie spirituelle constitue alors le moteur le plus puissant de l'adhésion ;

c) le recommençant, c'est-à-dire celui qui se ré-affilie à une religion qui était perçue jusque-là de façon purement extérieure, formelle.

En fait, plus que de conversion au sens défini ci-dessus, il serait peut-être préférable de parler d'adhésion croyante. On réserverait alors le vocable « conversion " au cas strict du passage d'une religion à une autre, le premier cas évoqué par Danièle Hervieu-Léger. Il faudrait préciser cependant que les sujets sont adultes, et que l'on ne saurait parler de conversion lorsqu'un individu déclarerait que sa pratique religieuse initiale (entendue au sens large) remonte à l'enfance. La problématique de la conversion, même telle qu'elle est définie dans le contexte contemporain, n'est donc peut-être pas tout à fait susceptible de décrire adéquatement « la religion en mouvement».

\subsection{D’une religion à l'autre}

Quoi qu'il en soit des définitions de la conversion, les catéchumènes ont eu connaissance de courants ou traditions religieuses. Les données révélées par le QA sont certainement sous-estimées. La figure du converti comme celui qui pense avoir

(15) Pierre Hadot, « Conversion », Encyclopaedia Universalis.

(16) Ibid. 1999.

(17) Danièle HERVIEU-LÉGER, Le pèlerin et le converti ; la religion en mouvement, Paris, Flammarion, 
enfin trouvé, au terme d'un long parcours, la source d'une vie spirituelle authentique est pourtant bien présente.

Il était demandé aux accompagnateurs s'ils avaient eu connaissance d'un parcours ou d'une imprégnation religieuse des catéchumènes avant leur arrivée : au total, $60 \%$ des dossiers retournés comportent une indication. La classification proposée aux enquêtés peut prêter à confusion, et certaines appellations font appel à la subjectivité des personnes. Il en va ainsi pour les "groupes sectaires ou ésotériques », pour les « protestants » ou les « évangélistes » quand ce n'est pas pour ce qui concerne le «paranormal». Sous réserve de ces quelques précautions, nous trouvons le détail suivant, présenté en deux tableaux complémentaires :

\begin{tabular}{|l|c|c|c|c|c|c|c|c|c|c|c|}
\hline Dénomination & Islam & Catholi & Paran. & Boudh & Ésotér & $\begin{array}{c}\text { Relig } \\
\text { trad }\end{array}$ & Judaïs. & $\begin{array}{c}\text { Fr. } \\
\text { Maç }\end{array}$ & Athée & $\begin{array}{c}\text { Scien- } \\
\text { tologie }\end{array}$ & Total \\
\hline Nombre & 47 & 37 & 26 & 14 & 10 & 9 & 8 & 2 & 2 & 1 & 156 \\
\hline$\%$ & $\mathbf{2 1 , 8}$ & $\mathbf{1 7 , 1}$ & $\mathbf{1 2}$ & $\mathbf{6 , 5}$ & $\mathbf{4 , 6}$ & $\mathbf{4 , 2}$ & $\mathbf{3 , 7}$ & $\mathbf{0 , 9}$ & $\mathbf{0 , 9}$ & $\mathbf{0 , 5}$ & $\mathbf{7 2 , 2}$ \\
\hline
\end{tabular}

Tableau 5 : nombre de mentions dans les $Q A$ de fréquentation d'une autre religion, secte ou groupe spirituel. Classement par ordre décroissant de fréquence.

Pourcentage calculé sur les $60 \%$ de $Q A$ retournés avec une mention.

\begin{tabular}{|l|c|c|c|c|c|c|}
\hline Dénomination & Évangélistes & Tém. Jého & Sectes & Anglicans & Baptistes & $\begin{array}{c}\text { Protestants } \\
\text { autres }\end{array}$ \\
\hline Nombre & 21 & 17 & 8 & 1 & 1 & 12 \\
\hline$\%$ & $\mathbf{9 , 7}$ & $\mathbf{7 , 9}$ & $\mathbf{3 , 7}$ & $\mathbf{0 , 5}$ & $\mathbf{0 , 5}$ & $\mathbf{5 , 6}$ \\
\hline
\end{tabular}

Tableau 5bis : nombre de mentions dans les $Q A$ de fréquentation d'un groupe affilié au protestantisme.

Telle qu'elle était formulée, la question portait sur une recherche consciente et adulte. Les résultats peuvent en être sujets à caution : comment imaginer que $22 \%$ des catéchumènes aient fréquenté adultes l'islam, ou bien $17 \%$ le catholicisme ? Il est plus vraisemblable que cette fréquentation ait été le cas d'une éducation en milieu musulman, catholique ou juif. Le catéchumène déclarerait à son accompagnateur, par exemple, que ses parents étant musulman pour l'un et protestant pour l'autre, il se serait trouvé livré à lui-même pour adhérer à l'une ou l'autre tradition religieuse. Ce qui concerne les grandes religions historiques (islam, judaïsme, protestantisme, catholicisme) semble impossible à interpréter sur la base de ces seules indications. Les religions traditionnelles relèvent d'une autre catégorie dans la mesure où la pratique de ces catéchumènes, vraisemblablement issus familialement d'autres continents, est difficile à apprécier.

Les autres «expériences religieuses», notamment celles qui relèvent de « groupes sectaires » ou de "paranormal », ne sont pas sans intérêt. Il faut en effet être adulte, dans la plupart des cas, pour être admis à les fréquenter. Au total, environ $40 \%$ des catéchumènes mentionnés par les accompagnateurs ont eu un parcours sur au moins un groupe religieux en tant qu'adultes, soit près de $25 \%$ de l'effectif total (correspondant au nombre de questionnaires retournés). Il est certain qu'une enquête plus fine révélerait des chiffres supérieurs. 


\section{2. Écritures, formation d'une mémoire?}

Les catéchumènes sont baptisés (eucharistiés et confirmés selon les cas) au terme de leur parcours de formation. Toutefois, cette échéance, précédée de quelques étapes, n'est pas automatique; des catéchumènes peuvent interrompre leur démarche en cours de route. La lettre de demande de baptême, adressée à l'évêque, revêt un caractère ambigu. À la fois rite de passage, examen d'entrée, récit de conversion, elle permet à tout le moins d'appréhender le degré d'acclimatation du catéchumène à ses accompagnateurs, pour ceux qui atteignent le terme de la formation. Le large éventail des capacités pratiques d'écriture qui se manifeste dans les lettres (de quelques lignes mal orthographiées à plusieurs pages) n'occulte pas le travail d'appropriation mémorielle. Il est cependant sélectif, selon des caractéristiques récurrentes d'un diocèse à l'autre.

L'analyse globale du panorama qui apparaît à travers les tableaux récapitulatifs doit mettre en évidence, en effet, que la différenciation entre province et région parisienne n'est pas un caractère déterminant. Selon les diocèses, des différences apparaissent certes dans la formulation des demandes de baptêmes. L'autonomie des responsables de catéchuménat diocésain est donc réelle : une formule identique de conclusion du parcours catéchuménal, la lettre de demande de baptême, conduit à des expressions distinctes. Pourtant, l'enquête sur la fréquence de vocables clés manifeste qu'une tendance lourde est à l'œuvre.

Les catéchumènes sont en mouvement. Ils ont glané dans leur formation les éléments qui deviennent autant de repères mémoriels jalonnant leur déplacement intérieur. L'émotion soulevée par cette appropriation semble authentifier ces expressions issues, semble-t-il, de la tradition catholique, mais participant peut-être d'abord d'une vulgate sociale recevable.

\subsubsection{De la difficile transmission d'une mémoire}

Les dénominations employées par les auteurs des lettres pour qualifier Dieu ne mentionnent que pour une part largement minoritaire la titulature proprement chrétienne. Tous diocèses confondus, le mot Dieu apparaît dans $79 \%$ des lettres, les mots liés à Jésus (en tant que Christ (18)) n'apparaissent que dans $41 \%$ des cas. Or, s'il faut en croire les déclarations, ce n'est pas faute d'avoir mis le Christ au premier plan dans la formation et les entretiens! Ne faut-il pas soupçonner alors une imprégnation du langage sur le divin par d'autres sources, vraisemblablement antérieures?

La transmission d'une mémoire neuve se réalise donc sous le mode de la superposition de strates mémorielles. La mention, dans un certain nombre de lettres, d'une antériorité implicite de l'expérience croyante (la foi était déjà au cœur de la personne ... à son dire) pose la question des références par lesquelles le sujet se découvre croyant. Sur quelles bases se structure l'expérience vécue d'une

(18) Il faut distinguer entre les mentions de Jésus, dont la valeur peut n'être qu'historique, et celles qui déterminent un caractère divin, comme Christ ou Jésus-Christ. Le mot Seigneur, qui évoque en général le Christ dans le langage interne de l'Église, est ambigu. La bible, à commencer par le premier Testament (Ancien testament), l'applique à Dieu. Il est impossible de statuer sur le sens qu'il revêt dans les lettres des catéchumènes. 
spiritualité, si ce n'est en prenant appui sur les catégories déjà disponibles de nomination du spirituel? Mais un déplacement s'opère grâce au catéchuménat (vocabulaire de la marche, du chemin), et l'évidence catholique s'introduit dans la vie croyante. Les prédicats déployés dans la formation catéchuménale sont loin, cependant, de s'imposer comme les seuls authentiques; ils coexistent avec ceux issus de la culture préalable de l'impétrant.

La lecture attentive des lettres établit la conviction que la fréquentation de la messe dominicale n'est pas la perspective dans laquelle sont inscrits les catéchumènes; il n'est donc pas vraisemblable d'augurer leur participation future et régulière. Les vocables associés (messe, eucharistie, communion) apparaissent dans $18 \%$ des lettres. Au fond, tout se passe comme si le modèle d'affirmation croyante actuellement majoritaire - catholiques non pratiquants - se diffusait dans des proportions approximativement semblables auprès des catéchumènes. En ce sens, il n’y a pas à s'étonner : comme l'énonce la sagesse populaire, "les chiens ne font pas les chats ».

Nous sommes donc amené à supputer que les modèles ecclésiologiques mis en œuvre par les catéchuménats diocésains et locaux ne sont pas suffisants pour déclencher une adhésion croyante pratiquante. Par là nous entendons aussi bien les enseignements structurants que les expériences vécues : vie de groupe, assemblées dominicales, etc. Il apparaît clairement, à travers les lettres, que la motion intérieure qui amène les catéchumènes vers l'Église catholique, et leur fait identifier leur dieu intime avec le Dieu chrétien, n'est pas relayée par l'Église elle-même. Elle ne semble pas capable de créer l'espace de vie qui permettrait à cette motion de prolonger son mouvement dans la vie du groupe chrétien (19).

Il en résulte que le catéchumène ne dispose pas, en règle générale, de formation à la vie chrétienne. Les raisons en sont sans nul doute multiples et complexes. L'origine et la formation des accompagnateurs y participe certainement; la vie des communautés chrétiennes, qui pourraient ne pas se comprendre elles-mêmes dans leur mystère propre est une autre cause.

\subsubsection{Mobilisés, mobiles}

\section{Émotion et chemin; les épreuves qualifiantes ?}

Le deuxième élément décisif contenu dans les lettres est le mouvement qui anime la vie intérieure des futurs catholiques. La découverte de Dieu s'accompagne ou se traduit dans la grande majorité des cas par un déplacement (63\% de mentions d'un vocable associé). Le vocabulaire du chemin rejoint étroitement l'autocompréhension de l'Église dans son affirmation la plus classique : une Église pérégrinante sur terre. Les catéchumènes s'affirment prêts à poursuivre ce chemin, qui les a rendus heureux.

Il faut cependant tempérer cette volonté exprimée, en tenant compte aussi de la forte valence émotionnelle attachée à l'objectif du baptême. Il n'est pas certain qu'un autre objectif puisse susciter une telle ferveur et libérer d'aussi puissantes forces intérieures pour surmonter l'obstacle du long terme.

(19) Le registre de la vie de groupe, de l'ecclésialité est représenté par les mots de la famille de : communauté, Église, famille. Ils apparaissent dans $56 \%$ des lettres. 
Le vocabulaire du chemin et celui de l'émotion, associés, peuvent être interprétés comme l'indice d'une épreuve qualifiante. L'adhésion au catholicisme serait alors le moyen de vérifier la pertinence de l'itinéraire spirituel vécu par l'individu. Parce qu'il est encore présent de façon massive, par ses monuments - lieux de mémoire nationale ? -, par sa diffusion générale dans la culture dès qu'il s'agit du divin, par sa plasticité accueillante - on s'arrange de toutes les situations -, le catholicisme offre la possibilité de constituer une mémoire croyante en paix avec une mémoire française. Le séisme démographique de son encadrement militant, la vaste restructuration de son déploiement géographique tendent à infirmer une telle hypothèse. Il ne faut pas cependant en exclure la pertinence pour un nombre de cas peut-être non négligeable, les conversions d'individus originaires d'autres continents.

\section{Pérégriner pour vivre}

Le modèle d'adhésion croyante est donc celui de la pérégrination : le catéchumène, en face de questions ou de difficultés dans la vie, cherche un chemin. Il n'est pas satisfait par les propositions auxquelles il a accès (athéisme, autre religion, incertitude de l'entourage, ...). À un moment donné se présente à lui la possibilité d'entrer en relation avec le Dieu catholique. Le contact avec un ou des membres de l'Église lui permet de parcourir un chemin intérieur, qui libère en lui beaucoup d'émotions. Sa vision de l'existence est transformée, quand ce n'est pas son comportement psychologique. Une structuration des connaissances et des émotions s'opère. Que cette pérégrination l'amène à rencontrer des gens sympathiques (et aussi compétents, ouverts, accueillants, etc.) ne le pousse pas automatiquement à s'inscrire dans le groupe des militants (les pratiquants); il lui suffit pour l'essentiel d'être compté au nombre de ceux qui s'en réclament. Du reste, on ne lui a pas vraiment fait comprendre quelle était la suite de l'itinéraire. S'il manifeste le désir de continuer, il ne donne aucun objectif ni but, même lorsque le diocèse présente la confirmation dans les années qui suivent.

Plus qu'une logique relevant du registre de la conversion, ce qui semble irriguer l'expérience des catéchumènes est une logique d'accomplissement. L'absence de vocabulaire concernant l'expérience du mal, qu'il s'agisse de la constellation mort/vie, ou lumière/ténèbres, sans parler de celle du péché montre qu'à aucun moment le catéchumène ne remet en cause sa manière de vivre. Il n'oppose pas un mode de l'erreur à une rectitude de vie. Le registre éthique est d'ailleurs pour ainsi dire inexistant $(8 \%)$. Il n'y a eu aucun mal commis - plutôt des souffrances et difficultés $(22 \%)$, qui trouvent leur résolution dans l'ouverture d'un chemin de certitudes questionnantes. En effet, le déplacement opéré est manifestement rassurant, il n'est pas une errance angoissante. La volonté exprimée de le poursuivre accrédite l'interprétation de la découverte spirituelle comme logique d'accomplissement de la personne. En ce sens, il relève pleinement de la modernité.

\section{Conclusion}

La sociologie des faits religieux souligne depuis de nombreuses années la convergence de phénomènes rencontrés dans diverses familles religieuses dès lors 
qu'elles sont confrontées à la modernité. Le concept de sécularisation, d'abord dominant puis peu à peu délaissé, cherche à rendre compte d'une érosion du religieux dans les sociétés occidentales, et tout particulièrement en France. Les différents réveils religieux, qui ont affecté depuis une trentaine d'années les grandes religions institutionnelles (catholicisme, protestantismes, judaïsme, etc.) ont conduit à réévaluer l'apparente inexorabilité du déclin de la religion.

En revanche, l'individualisation croissante des pratiques religieuses est une caractéristique largement répandue dans les sociétés de la modernité. L'enquête menée ici n'infirme en rien le constat. Comment penser alors la socialisation des individus aux contenus de croyances? Comment l'Église catholique en France s'efforce-t-elle de prendre en charge le phénomène, tant son idéal de communion et d'organisation autour d'un clergé structuré - par ailleurs menacé par l'effondrement démographique - contrevient à la fluence des identités religieuses ?

Les nouveaux catholiques n'échappent pas aux tendances de fond qui régissent les comportements observables des identités religieuses en France. On peut en conclure que le système catholique français fonctionne globalement en phase avec les attentes de la société moderne. Il n'est pas fait violence au désir d'accession personnalisée au religieux. Comment évaluer un tel résultat? Deux réflexions sont possibles. D'une part, on pourrait conclure à la fin d'un modèle catholique de conformité et de mise aux normes de la vie chrétienne. Le mouvement de réforme commencé dès le $\mathrm{XIX}^{\mathrm{e}}$ siècle en matière d'unification des règles liturgiques (extension du modèle romain de célébration de la messe) et de la codification canonique (Codes de 1917 et de 1983) trouverait ici ses limites, au sein de cette modernité qui l'a vu naître.

Mais il est également possible de relever la convergence très forte entre l'individualisation de la formation chrétienne et l'accent mis depuis la Contre-Réforme sur la personnalisation de la conviction croyante (à travers la spiritualité notamment). Les catéchumènes ne seraient pas tant formés par le collectif que sur le modèle de la direction spirituelle classique.

Ce profil moderne des convertis au catholicisme ne risque-t-il pas de se maintenir, et leur itinérance de se poursuivre? Peuvent-ils se satisfaire d'une adhésion qui les a enthousiasmés pour le temps qu'ils passent à leur formation, mais qui risque fortement de ne pas susciter le même engouement après la réception des trois sacrements de la vie chrétienne? Autrement dit, l'enthousiasme dont font preuve les catéchumènes porte-t-il sur le dispositif d'accompagnement ou bien sur le contenu et les perspectives ultérieures dont le dispositif n'est que l'antichambre?

Posée en termes sociologiques, la question du devenir des nouveaux catholiques renvoie donc aux interactions propres entre les membres du groupe ecclésial : s'agit-il d'interactions closes ou ouvertes ? Quel degré de conformité au groupe estil requis de la part des impétrants ? La normalisation nécessaire est-elle compatible avec la demande de ceux qui veulent participer au groupe ? Il revient à ce dernier, et au premier chef à ses responsables, de définir les règles d'accès et de vie ordinaire. De ce point de vue, l'enquête montre que le discours qui porte sur les Sacrements de l'Initiation n'est pas reçu comme tel par les candidats.

L'organisation du catéchuménat met en lumière la profonde mutation de l'Église catholique en France. L'encadrement largement assuré par des fidèles laïques associé à l'individualisation de la formation à la vie chrétienne modifient le 
rapport à l'autorité. Le modèle qui s'annonce depuis une dizaine d'années (durée de vie établie des catéchuménats) repose sur une diffusion de la compétence chrétienne par le bouche-à-oreille de l'entretien familier (20). Les clercs ne sont certes pas absents du fonctionnement - la célébration liturgique des étapes de baptême le prouve. À moins cependant de supposer que les entretiens individuels suivent des directives émises par les curés, tout laisse à penser que le discours qui fait autorité est celui de l'accompagnateur. Bref, le modèle de la direction spirituelle de l'époque classique est pris en charge par les laïcs, et le subvertit donc complètement; le clerc n'est plus le référent, il cède la place au laïc.

En conséquence, la diffusion des normes ecclésiales de comportement, tant du point de vue éthique que liturgique, s'opère de façon diffuse. On pourrait parler à cet égard d'une "normalisation émiettée » : une diffusion continue par un corps informel de laïcs du corpus de croyances et de normes catholiques s'opère dans l'Église en France. Les dizaines de milliers de nouveaux catholiques qui ont été agrégés à la vie chrétienne depuis plus de dix ans ont reçu les enseignements d'accompagnateurs présents continûment.

Cette normalisation émiettée aura des conséquences sur la cohésion de la mémoire - réelle ou mythique - des groupes catholiques français. Un mixte de repères puisés dans le bricolage permanent des cultures, par les médias, les modes de vie urbains ou les immigrations diverses, se surajoute aux normes et souvenirs issus de la Tradition de l'Église catholique. L'avenir dira si la transmission de la conscience catholique française, dans son rapport à la société laïque et au politique, notamment, laissera place à une recomposition métisse : défi pour le catholicisme romain aussi bien que pour la République.

\author{
Nicolas de BREMOND D'ARS \\ CEIFR-EHESS
}

(20) Ironie de l'histoire : « entretien familier » est la signification étymologique de l'homélie, communément comprise comme le «sermon du curé »! 
Résumé

Cinquante mille personnes ont fréquenté l'Église catholique en France depuis dix ans, en vue d'y adhérer. Une enquête menée par le Service National du Catéchuménat, en lien avec le Centre d'Études Interdisciplinaires des Faits Religieux, montre que l'afflux de ces candidats soumet l'environnement catholique paroissial à une tension critique de trois côtés : par la mouvance des territoires et des niveaux d'appartenance catholique, par l'individualisation de la transmission et par la modification du dispositif d'autorité. Elle révèle en outre une certaine inadéquation entre la formation donnée par les services locaux de catéchuménat et les pratiques et normes des communautés catholiques existantes. De ce fait, une importante modification des modalités de transmission de la mémoire catholique - rites, pratiques et contenus de croyances est initiée. Elle est perceptible à travers les lettres que les catéchumènes écrivent à la fin de leur parcours d'initiation en vue de demander le baptême. On a à faire à une normalisation émiettée, qui prend le pas sur la socialisation collective classique, encore présente au niveau de l'éducation des enfants.

Abstract

Over the last ten years fifty thousand people have frequented the Catholic Church in France with the intention of joining it. A survey carried out jointly by the Service National du Catéchumenat (National Service for Religious Education) and the CEIFR (Center for the Interdisciplinary Study of the Religious Factor) indicates that the influx of these candidates has submitted the Catholic parish environment to a critical tension from three different angles: the fluidity of the Catholic geography and of the degrees of belonging to the Church; the individualization of the transmission process and the transformation of the system of authority. The survey highlights as well a certain inadequacy between the education given by the local services for the catechumens and the practices and norms of the existing Catholic communities. An important modification of the modalities of transmission of Catholic memory - rites, practices, and beliefs - is thereby initiated. It is perceptible in the letters that the catechumens write at the end of the period of initiation they follow before being baptized. We are witnessing a fragmented normalization which is taking over from the classic process of collective socialization still present at the level of the education of children.

Resumen

Desde hace diez años, cincuenta mil personas asistieron a la Iglesia católica en Francia con la intención de adherir a ella. Una encuesta llevada a cabo por el Servicion Nacional de Catecumenado, ligado con el CEIFR, muestra que la llegada de estos candidatos somete al medio ambiente católico parroquial a una triple tensión crítica : a causa de la movilidad de los territorios y de los niveles de pertenencia católica, por la individualización de la transmisión y por la modificación del dispositivo de autoridad. Revela, además, una cierta inadecuación entre la formación dada por los servicios locales de catecumenado y las prácticas y normas de las comunidades católicas existentes. A partir de este hecho, una importante modificación de las modalidades de transmisión de la memoria católica - ritos, prácticas, y contendidos de creencia - ha comenzado. Es posible leerla en lac cartas que los catecúmenos escriben hacia el final de su recorrido de iniciación para pedir el bautismo. Estamos frente a una normalización fragmentada, que se impone a una socialización colectiva clásica, aún presente en el nivel de la educación de los niños. 